\title{
Cytomorphological study of thyroid carcinoma
}

\author{
IULIANA MOHOREA ${ }^{1,2}$, DANA TERZEA ${ }^{3}$, DANIELA MIHALACHE ${ }^{2,4}$, \\ BOGDAN SOCEA $^{5,6}$, DRAGOŞ ŞERBAN ${ }^{5,7}$ and MIHAI CEAUSU ${ }^{1}$
}

\author{
${ }^{1}$ Department of Pathology, 'Carol Davila' University of Medicine and Pharmacy, 020021 Bucharest; \\ ${ }^{2}$ Department of Pathology, Braila Emergency County Hospital, 810325 Braila; ${ }^{3}$ Department of Pathology, \\ 'C.I. Parhon' Clinical Hospital for Endocrine Diseases, 011863 Bucharest; ${ }^{4}$ Department of Pathology, Faculty of Medicine \\ and Pharmacy, 'Dunarea de Jos' University of Galati, 800008 Galati; ${ }^{5}$ Department of Surgery, 'Carol Davila' University of \\ Medicine and Pharmacy, 020021 Bucharest; ${ }^{6}$ Department of Surgery, 'Sf. Pantelimon' Emergency Clinical Hospital, \\ 021659 Bucharest; ${ }^{7}$ Department of Surgery, University Emergency Hospital, 050098 Bucharest, Romania
}

Received September 3, 2021; Accepted October 5, 2021

DOI: $10.3892 / \mathrm{etm} .2021 .11040$

\begin{abstract}
The most common neoplasm of the endocrine system is found in the thyroid gland with a significant increase in recent decades largely due to modern diagnostic methods. Thyroid tumors generally have a favorable evolution, but there are also aggressive variants with a poor prognosis. In these aggressive tumors, the most reliable method of detecting and making a differential diagnosis is represented by ultrasound-guided fine-needle cytopuncture, confirmed by histopathological examination. Although fine-needle aspiration puncture and cytological examination are considered to have a high sensitivity and specificity, diagnostic certainty is established later only by histopathological examination. Fine-needle aspiration cytopuncture of the thyroid gland correlated with histopathological examination has played a crucial role in recognizing and identifying variants of papillary carcinoma known to have aggressive biological behavior, especially in cases of poorly differentiated carcinoma. Recognition of aggressive variants of papillary carcinoma is of major importance in the prognosis and clinical management of patients. The aim of this study was to present the correlations found in a series of thyroid tumors from patients treated in surgery and oncology departments, as well as tumors accidentally detected during autopsies in the department of forensics. All the cases selected in the study benefited from a complex histopathological diagnosis adapted to each case in order to ensure maximum efficiency.
\end{abstract}

Correspondence to: Dr Bogdan Socea, Department of Surgery, 'Carol Davila' University of Medicine and Pharmacy, 37 Dionisie Lupu Street, 020021 Bucharest, Romania

E-mail: bogdan.socea@umfcd.ro

Key words: thyroid carcinoma, thyroid cytomorphology, fine-needle aspiration, cytopuncture of the thyroid, histopathology, thyroid tumors

\section{Introduction}

Thyroid nodules are quite common in the general population. The cases mentioned in the medical publications report a prevalence of $50 \%$ of thyroid nodules detected during autopsies in unknown subjects with thyroid pathology. Malignant nodules are found in approximately $5 \%$ of the population (1-5).

Thyroid cancer accounts for $1 \%$ of all malignancies, but is increasingly becoming more common worldwide. Thyroid carcinoma affects women more often than men, in most cases affecting patients aged between 25 and 65 years. The annual incidence of thyroid tumors varies depending on age, sex, race, geographic region as well as hormonal and environmental factors $(6,7)$.

The gold standard in the early diagnosis of thyroid tumors is considered to be fine-needle aspiration biopsy of thyroid nodules, ultrasound-guided, followed by cytological evaluation. It is a simple, safe method, which involves minimal costs and has a favorable diagnostic accuracy (8-11).

Huang et al presented this method as having a sensitivity and specificity of approximately 83 and $92 \%$ with a failure rate between 1 and $21 \%$, emphasizing that these failure rates appear because of the technique and experience of execution (12).

The Bethesda System for Reporting Thyroid Cytopathology standardized the results obtained through fine-needle aspiration procedure and facilitated communication and collaboration between physicians (13). Gupta et al (14) and Miftari et al (15) concluded that the fine-needle aspiration technique can provide clear evidence in the diagnosis of aggressive variants of thyroid papillary carcinoma $(14,15)$.

The surgical treatment comprises primarily of total thyroidectomy with laryngeal recurrent nerve protection (16). Thus, the aim of the present study was to determine the correlations of thyroid tumors obtained from patients and those harvested during autopsies. A complex histopathological diagnosis is imperative in tailoring to each case for maximum efficiency. 


\section{Materials and methods}

Case selection for the study batch. The cases included in the present study came from thyroids harvested during autopsies, from patients operated in the surgery wards and from the patients registered in the oncology ward, from the Braila Emergency County Hospital.

The study batch was composed of 442 males (78\%) and 119 females $(22 \%)$ (sex ratio $=3.71$ ), with age ranging from 10 to 94 years (mean $=60.32, \mathrm{SD}= \pm 15.42$ ). The highest incidence was found in patients aged between 60 and 70 years. The inclusion criteria for the study were: patients presenting thyroid tumors, treated in the surgery and oncology departments, as well as thyroid tumors accidentally detected during autopsies in the Department of Forensics from the Braila Emergency County Hospital in the selected period of time. None of the subjects with accidentally detected tumors had evidence of thyroid disease. Patients that had no histopathologically confirmed diagnostic were excluded. The urban/rural distribution encountered in patients with thyroidian neoplasms was approximately equal.

The following types of interventions were performed on the thyroid gland: fine-needle aspiration technique and cytological examination, biopsy (in case of non-removable tumor), unilateral lobectomy with isthmusectomy, subtotal thyroidectomy, unilateral lobectomy with isthmusectomy and contralateral subtotal lobectomy, total thyroidectomy, and extended thyroidectomy in the pre-thyroid muscles. Thyroid nodules $<1 \mathrm{~cm}$ in diameter were generally not punctured, unless they showed suspicious ultrasound-detected changes such as micro-calcifications.

The study was approved by the Ethics Committee of the County Emergency Hospital Braila (Braila, Romania), approval no. 37948/08.10.2020, respecting the ethical standards of the 1975 'Helsinki Declaration', revised in 2000, and complying with the national legislation. Written informed consent was obtained from the patients.

Tissue sampling and staining. The harvested thyroid specimens were fixed in $10 \%$ formalin, buffered for $24 \mathrm{~h}$ and in the case of bulky goiter, several incisions were performed to facilitate fixation. The thyroid gland was dissected and carefully separated from the soft tissues of the peri-thyroid space, fixed in $10 \%$ formalin and then weighed and measured.

Macroscopic changes in the observed structure were noted, especially the whitish star-shaped scarred areas, suspected of being carcinomas. Multiple systematic cuts were performed to cover a wide range of undetected pathologies in the macroscopic examination.

After weighing the thyroidectomy section, 2- to 3- $\mu \mathrm{m}$ sections were cut to allow complete visualization of the thyroid capsule and detection of infra-centrimetric carcinomas.

Over 1,000 sections of thyroid tissue were sampled. The selected tissue samples were fixed in 10\% neutral-buffered formalin $(\mathrm{pH}$ 7.0) for 24-48 hat room temperature and paraffin embedded. The sections were cut at $5 \mu \mathrm{m}$ and stained with standard hematoxylin and eosin at room temperature for $2 \mathrm{~h}$.
The samples harvested from the thyroid gland by fine-needle aspiration were processed as smears. The slides were fixed in 95\% alcohol and stained with Papanicolaou. Then, the slides were subjected to complex cyto-histopathological examination.

All the slides were examined and photographed with a Nikon Eclipse Ci. Digital images captured using a Digital Microscope Camera program were processed and analyzed with the Photos App, running under Windows 10.

Statistical analysis. Variable categories were summarized as counts, percentages, and continuous variables such as medians. Data are presented as mean \pm SD. Statistical comparisons were made using the Minitab ${ }^{\circledR}$ program (version 19, Trialware TM), which showed a mean value of 60.32 for the age (SD, 15.42). The Pearson Chi-square test was used to compare the type, size of the tumor, sectioning intervals $(0.5 \mathrm{~cm}$ for papillary microcarcinomas vs. $1-3 \mathrm{~mm}$ as the value for latent papillary carcinomas), multifocality, lymph node metastases.

The following indicators were calculated in the statistical analysis: median, mean, standard deviation, asymmetry and vault indicators for the analyzed immunohistochemical parameters. $\mathrm{P}<0.05$ was considered statistically significant in all our tests. There were no repeated experiments.

\section{Results}

Following the 526 autopsies performed in a time interval of 3 years (January 2017-January 2020), 51 thyroid tumors were identified, 153 cases with thyroid nodules, of which 135 were multinodular goiters and 18 uninodular goiters.

A further 17 cases were selected from the histopathological registers between December 2016 and December 2020 from patients operated in the surgery departments and also patients recorded in the oncology department of the County Emergency Hospital Braila, who presented thyroid tumors with aggressive forms.

A total of 68 cases with thyroid tumors were included in the study. From these, 60 were papillary carcinomas (Figs. 1-3), 4 follicular carcinomas, two poorly differentiated (Fig. 4), one medullary (Fig. 5) and one squamous (Fig. 6). Patients included in surgical treatments also benefited from cytological diagnosis by fine-needle aspiration.

Papillary carcinomas were carefully reanalyzed to determine the histological subtype. Microcarcinomas were identified in the vast majority of cases (55 cases), but there were also 5 cases with variants of papillary carcinoma with aggressive potential, of which included: tall cell variant ( 2 cases), one case with diffuse sclerosis, one hobnail variant and one diffuse follicular variant.

We used the criteria of the World Health Organization (WHO) to diagnose and classify the histological subtypes of the thyroid tumors (17).

Carcinomas that were $<1 \mathrm{~cm}$ in size were classified, according to the definition, as papillary microcarcinomas. These were detected incidentally during the autopsies. The patients had a long evolution, without symptoms, their detection being established because of the thorough and complex analysis of the thyroid gland. 


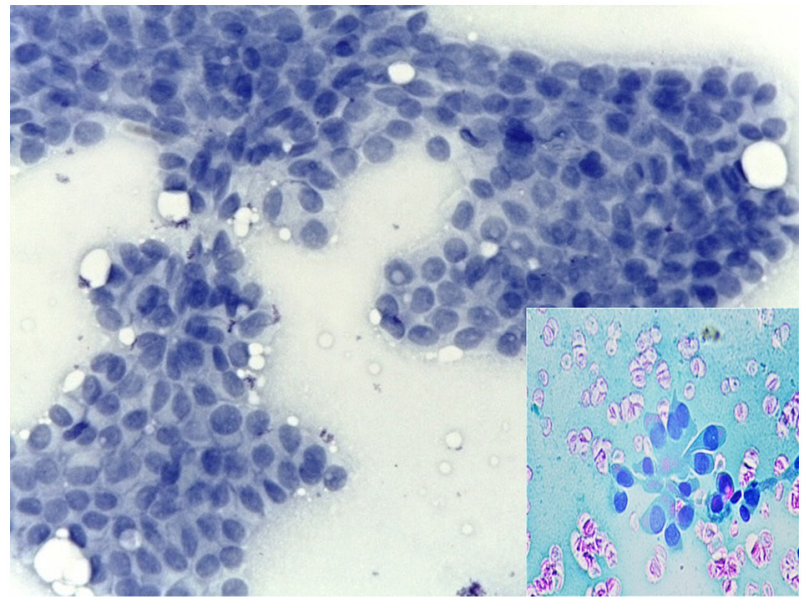

Figure 1. Papillary thyroid carcinoma. Papillary thyroid carcinoma identified using cytology and PAP. Inset: papillary carcinoma showing tall cell variant; magnification, $\mathrm{x} 400$.

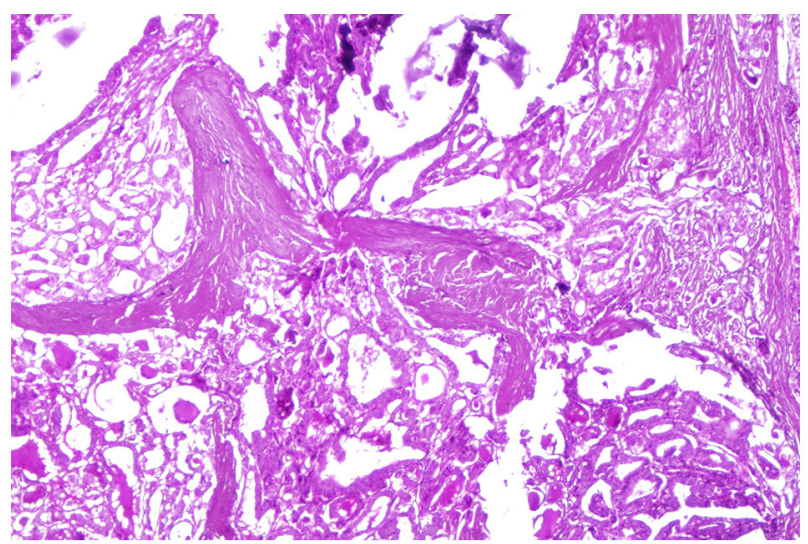

Figure 2. Papillary thyroid carcinoma. Follicular variant with micro-calcifications identified via hematoxylin and eosin; magnification, x400.

\section{Discussion}

By definition, aggressive variants of papillary thyroid carcinoma have the same characteristic nuclear features (nuclear inclusions, nuclear notches) with papillary carcinoma but have different architectural arrangements and distinct cellular features.

The tall-cell variant of papillary carcinoma was diagnosed in two male patients, aged 78 and 76 years, respectively, where the variant showed a characteristic appearance since the diagnostic cytopuncture, subsequently confirmed by histological examination.

Leung et al (18) suggested that this variant of tall-cell papillary carcinoma could not be diagnosed cytologically by fine-needle aspiration technique. However, Guan et al (19) and Das et al (20) showed that the tall-cell variant was correctly recognized in cytological specimens in 30-100\% of cases.

The hobnail variant of papillary carcinoma was detected in a 69-year-old man who showed symptoms of local pain in the cervical region, dyspnea, dysphonia and dysphagia. The fine-needle aspiration method showed typical morpho-cytological features: the nuclei were located at the top of the cell

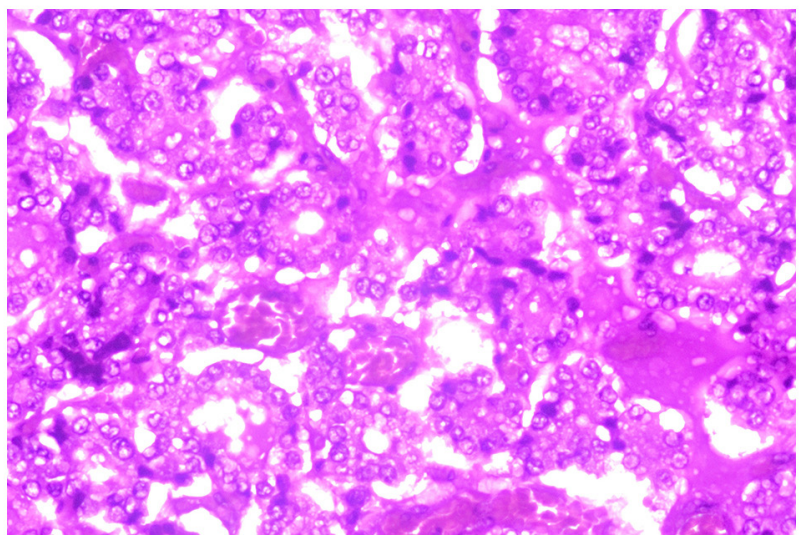

Figure 3. Papillary thyroid carcinoma. Details with optical clear nuclei and visible nucleoli. Identified using hematoxylin and eosin; magnification, x200.

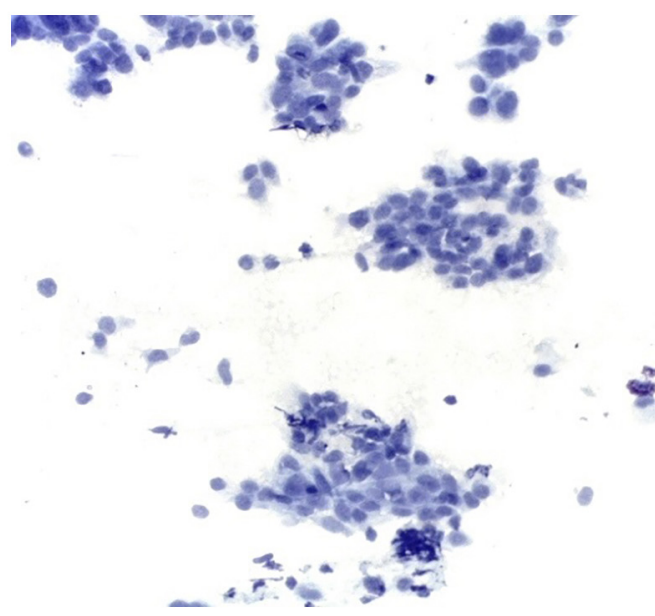

Figure 4. Poorly differentiated thyroid carcinoma. Poorly differentiated thyroid carcinoma identified using cytology and PAP; magnification, x200.

or in the middle of the cytoplasm, giving the cell the typical hobnail appearance $(21,22)$.

The diffuse sclerosing variant of the papillary carcinoma was identified in a 43-year-old patient. In the present study, aspiration puncture used in the diffuse sclerosing variant showed a smear with moderate-high cellularity, with the presence of colloid dispersed in the background. The characteristic cytological features highlighted the arrangement of the cells in three-dimensional layers, ball-like groups and cohesive groups of cells mixed with inflammatory cells. These characteristics are similar to those mentioned in the literature.

The diffuse follicular variant is defined as having a characteristic diffuse spreading in the thyroid gland with pseudo-areas of follicular patterns and nuclear features that are characteristic for classical thyroid papillary carcinoma. The diffuse follicular variant was identified in a 46-year-old woman who came with dyspnea, dysphagia, dysphonia and vocal cord paresis. Diagnostic cytopuncture and histological examination revealed a pattern of follicular growth and nuclear features characteristic of classical papillary thyroid carcinoma (23).

Thyroid follicular carcinoma occurred in 4 cases in the study group. Of these, three were women and one was a man, 


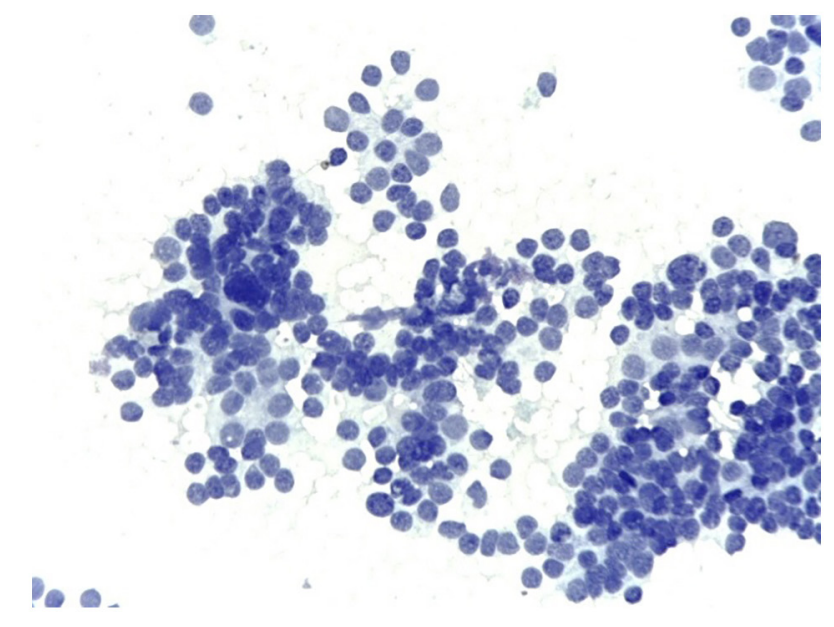

Figure 5. Medullary thyroid carcinoma. Medullary thyroid carcinoma identified using cytology and PAP; magnification, $\mathrm{x} 400$.

all 4 cases, aged between 50 and 60 years. Follicular thyroid carcinoma occurs in $10-15 \%$ of cases with thyroid malignancies and can also have an invasive behavior (24). In the present study, aspiration puncture showed a morphology that was close to normal, with a round nucleus, increased in volume, chromatin arranged marginally, without clarifications, but with an increased number of mitoses. One case consisted mainly of Hurthle cells with small cell microfollicular architecture, intensely eosinophilic, vesicular cytoplasm, with small, uniform, round nuclei centered by prominent nucleoli. Poorly differentiated carcinoma was found in 2 cases of women aged 76 and 83 years, respectively.

In the present study, fine-needle aspiration detected high cellularity with the presence of groups of agglomerated cells showing an increased nucleus/cytoplasmic ratio with variable nuclear atypia, mitotic figures, chromatin, fine granular, with distribution in salt and pepper. Poorly differentiated carcinoma are frequently widely invasive, with infiltration of perithyroid tissues observed in 60-70\% of the cases presented.

The cytopathological diagnosis presented in extensive studies could be specified only in $5-14 \%$ of cases. The remaining cases were diagnosed as 'suspicion of follicular neoplasm' or carcinomas (other than papillary carcinoma, follicular variant of papillary carcinoma or without other specifications) $(25,26)$.

Medullary carcinoma can exist in two variants: sporadic and familial. The familial variant has an autosomal dominant inheritance, involving the mutation of the proto-oncogene RET (24). Thyroid medullary carcinoma was detected in a 52-year-old woman who clinically presented with a hard, painful nodule at the cervical level accompanied by flush-like symptoms and diarrhea and paraclinically high levels of plasma serum calcitonin.

Diagnostic cytopuncture revealed a solid proliferation composed of round and polygonal cells with amphophilic cytoplasm and medium-sized nuclei, separated by high vascular stroma, hyalinized with amyloid deposition, with carcinoid-like growth pattern and the presence of amyloid deposits.

Squamous cell carcinoma of the thyroid gland is a very rare entity, the WHO falling under the subchapter

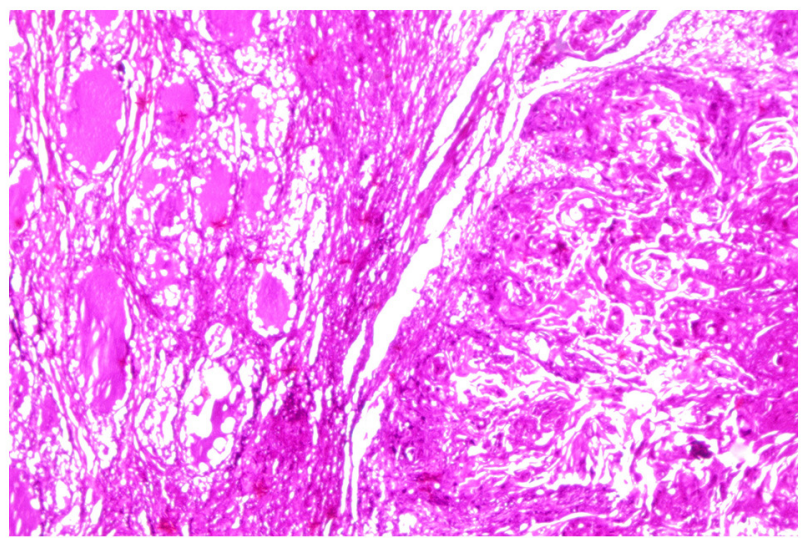

Figure 6. Squamous cell carcinoma. Squamous cell carcinoma of the thyroid, identified using hematoxylin and eosin; magnification, $\mathrm{x} 400$.

'other carcinomas' in the category of malignant epithelial tumors (27-29). Squamous thyroid carcinoma was diagnosed in a 72-year-old woman who presented with a hoarse voice, dysphagia and cachexia. External examination of the neck region revealed a tumor mass plunging retrosternally. Fine-needle aspiration revealed the presence of colloid and malignant cells.

Cytomorphological characteristics correlated with clinical data, immunohistochemical markers and the molecular profile can quickly elucidate complex and difficult cases. In our study batch, the patients with aggressive variants, who were preoperatively diagnosed by fine-needle aspiration, received surgery with extensive dissection in the neck region. They benefited from imaging scans to rule out the presence of metastases and were included more rapidlyin national oncology programs. Moreover, these patients required close supervision and monitoring, and in advanced cases of the disease they benefited from complex palliative care.

On some cytological smears it was not possible to specifically diagnose an aggressive histological subtype of thyroid carcinoma. However, even in these cases, the visualized morphological characteristics were reported, signaling the possibility of the existence of an aggressive variant of thyroid carcinoma. Despite the increasing incidence, thyroid cancer mortality has been decreasing in recent decades due to an improved diagnostic process and thyroid treatment strategy expected with the development of knowledge in the field.

In conclusion, recognition of aggressive variants of thyroid carcinoma requires the application of more aggressive surgical treatment from the beginning with the optimization and minimization of post-therapeutic sequelae and the avoidance of further surgeries. It is also important for a cytopathologist to have experience and be familiarized with the cytomorphological features encountered in poorly differentiated carcinomas and aggressive variants of thyroid carcinomas to avoid misdiagnosis with other types of primary or secondary thyroid tumors.

\section{Acknowledgements}

Not applicable. 


\section{Funding}

No funding was received.

\section{Availability of data and materials}

The datasets used and/or analyzed during the current study are available from the corresponding author on reasonable request.

\section{Authors' contributions}

IM and DT performed the histological and cytological examinations, and had major contributions in writing the manuscript. IM and DM analyzed and interpreted the patient data. BS, DS and $\mathrm{MC}$ searched the literature for similar work and articles and contributed to writing the manuscript. All authors read and approved the final manuscript. All authors confirm the authenticity of the data and the paper.

\section{Ethics approval and consent to participate}

The study was conducted according to the World Medical Association Declaration of Helsinki. The protocol was approved (no. 37948/08.10.2020) by the local Bioethics Committee from the Brăila Emergency County Hospital (Braila, Romania). All patients previously signed a written informed consent about hospitalization, treatment and a possible future publication of data.

\section{Patient consent for publication}

Not applicable.

\section{Competing interests}

The authors declare that they have no competing interests.

\section{References}

1. Ramirez-Gonzalez LR, Sevilla-Vizcaino R, Monge-Reyes P, Aldaz-Dorantes JE, Marquez-Valdez AR, Garcia-Martinez D, Gonzalez-Ojeda A and Fuentes-Orozco C: Findings of thyroid nodules in autopsies in Western Mexico. Rev Med Inst Mex Seguro Soc 55: 594-598, 2017 (In Spanish).

2. Vaideeswar P, Singaravel S and Gupte P: The thyroid in ischemic heart disease: An autopsy study. Indian Heart J 70 (Suppl 3): S489-S491, 2018.

3. Davies L, Morris LG, Haymart M, Chen AY, Goldenberg D, Morris J, Ogilvie JB, Terris DJ, Netterville J, Wong RJ, et al: American association of clinical endocrinologists and American college of endocrinology disease state clinical review: The increasing incidence of thyroid cancer. Endocr Pract 21: 686-696, 2015.

4. Milas Z, Shin J and Milas M: New guidelines for the management of thyroid nodules and differentiated thyroid cancer. Minerva Endocrinol 36: 53-70, 2011.

5. Mohorea IS, Socea B, Şerban D, Ceausu Z, Tulin A, Melinte V and Ceausu M: Incidence of thyroid carcinomas in an extended retrospective study of 526 autopsies. Exp Ther Med 21: 607, 2021

6. Kaliszewski K, Zubkiewicz-Kucharska A, Kiełb P, Maksymowicz J, Krawczyk A and Krawiec O: Comparison of the prevalence of incidental and non-incidental papillary thyroid microcarcinoma during 2008-2016: A single-center experience. World J Surg Oncol 16: 202, 2018.

7. Slijepcevic N, Zivaljevic V, Marinkovic J, Sipetic S, Diklic A and Paunovic I: Retrospective evaluation of the incidental finding of 403 papillary thyroid microcarcinomas in 2466 patients undergoing thyroid surgery for presumed benign thyroid disease. BMC Cancer 15: 330, 2015 .
8. Park SY, Jung YS, Ryu CH, Lee CY, Lee YJ, Lee EK, Kim SK, Kim TS, Kim TH, Jang J, et al: Identification of occult tumors by whole-specimen mapping in solitary papillary thyroid carcinoma. Endocr Relat Cancer 22: 679-686, 2015.

9. Lee YS, Lim H, Chang HS and Park CS: Papillary thyroid microcarcinomas are different from latent papillary thyroid carcinomas at autopsy. J Korean Med Sci 29: 676-679, 2014.

10. Janovsky CCPS, Bittencourt MS, Novais MAP, Maciel RMB, Biscolla RPM and Zucchi P: Thyroid cancer burden and economic impact on the Brazilian public health system. Arch Endocrinol Metab 62: 537-544, 2018.

11. Miyauchi A, Ito Y and Oda H: Insights into the management of papillary microcarcinoma of the thyroid. Thyroid 28: 23-31, 2018.

12. Huang LY, Lee YL, Chou P, Chiu WY and Chu D: Thyroid fine-needle aspiration biopsy and thyroid cancer diagnosis: A nationwide population-based study. PLoS One 10: e0127354, 2015.

13. Rossi ED, Faquin WC and Pantanowitz L: Cytologic features of aggressive variants of follicular-derived thyroid carcinoma. Cancer Cytopathol 127: 432-446, 2019.

14. Gupta S, Sodhani P, Jain S and Kumar N: Morphologic spectrum of papillary carcinoma of the thyroid: Role of cytology in identifying the variants. Acta Cytol 48: 795-800, 2004.

15. Miftari R, Topçiu V, Nura A and Haxhibeqiri V: Management of the patient with aggressive and resistant papillary thyroid carcinoma. Med Arch 70: 314-317, 2016.

16. Alecu L, Slavu I, Tulin A, Braga V, Socea B and Nitipir C: A current view on recurrent laryngeal nerve injury in total thyroidectomy. Mod Med 28: 7-12, 2021.

17. Lloyd RV, Osamura RY, Klöppel G and Rosai J (eds): WHO Classification of Tumours of Endocrine Organs. Vol 10.4th edition. International Agency for Research on Cancer, Lyon, 2017.

18. Leung AK, Chow SM and Law SC: Clinical features and outcome of the tall cell variant of papillary thyroid carcinoma. Laryngoscope 118: 32-38, 2008.

19. Guan H, Vandenbussche CJ, Erozan YS, Rosenthal DL, Tatsas AD, Olson MT, Zheng R, Auger M and Ali SZ: Can the tall cell variant of papillary thyroid carcinoma be distinguished from the conventional type in fine needle aspirates? A cytomorphologic study with assessment of diagnostic accuracy. Acta Cytol 57: 534-542, 2013.

20. Das DK, Mallik MK, Sharma P, Sheikh ZA, Mathew PA, Sheikh M, Mirza K, Madda JP, Francis IM and Junaid TA: Papillary thyroid carcinoma and its variants in fine needle aspiration smears. A cytomorphologic study with special reference to tall cell variant. Acta Cytol 48: 325-336, 2004.

21. Sheu SY, Schwertheim S, Worm K, Grabellus F and Schmid KW: Diffuse sclerosing variant of papillary thyroid carcinoma: Lack of BRAF mutation but occurrence of RET/PTC rearrangements. Mod Pathol 20: 779-787, 2007.

22. Lee SH, Jung CK, Bae JS, Jung SL, Choi YJ and Kang CS: Liquid-based cytology improves preoperative diagnostic accuracy of the tall cell variant of papillary thyroid carcinoma. Diagn Cytopathol 42: 11-17, 2014.

23. Gupta S, Ajise O, Dultz L, Wang B, Nonaka D, Ogilvie J, Heller KS and Patel KN: Follicular variant of papillary thyroid cancer: Encapsulated, nonencapsulated, and diffuse: Distinct biologic and clinical entities. Arch Otolaryngol Head Neck Surg 138: 227-233, 2012.

24. Humphrey PA, Dehner LP, Pfeifer JD, Agarwal A and Al-Kateb H: The Washington Manual of Surgical Pathology. 2nd edition. Lippincott Williams \& Wilkins, Philadelphia, pp.410-416, 2012.

25. Bongiovanni M, Sadow PM and Faquin WC: Poorly differentiated thyroid carcinoma: A cytologic-histologic review. Adv Anat Pathol 16: 283-289, 2009.

26. Kane SV and Sharma TP: Cytologic diagnostic approach to poorly differentiated thyroid carcinoma: A single-institution study. Cancer Cytopathol 123: 82-91, 2015.

27. Lam AK: Squamous cell carcinoma of thyroid: A unique type of cancer in World Health Organization classification. Endocr Relat Cancer 27: R177-R192, 2020.

28. Hedinger C, Williams ED and Sobin LH (eds): Other carcinomas. In: Histological Typing of Thyroid Tumours (World Health Organ. International Histological Classification of Tumours), 2nd edition, pp. 14-15. Springer, Germany, 1988.

29. Lam AK: Pathology of endocrine tumors update: World health organization new classification 2017 - other thyroid tumors. AJSP: Rev Rep 22: 209-216, 2017.

This work is licensed under a Creative Commons Attribution-NonCommercial-NoDerivatives 4.0 International (CC BY-NC-ND 4.0) License. 